\title{
Shoulder impingement syndrome in water polo players: muscle shortening manoeuvre controls pain intensity, recovers function and normalizes sonographic parameters
}

\author{
Diego Longo ${ }^{1,2}$ (D) Enrico Branchi ${ }^{1}$ - Pietro Matucci-Cerinic ${ }^{3} \cdot$ Maria Angela Bagni ${ }^{1} \cdot$ Marco Matucci-Cerinic $^{1,4,5}$. \\ Daniela Melchiorre ${ }^{1,5}$
}

Received: 28 September 2021 / Accepted: 29 November 2021

(c) Società Italiana di Ultrasonologia in Medicina e Biologia (SIUMB) 2022

\begin{abstract}
Purpose To evaluate the effects of muscle shortening manoeuvre (MSM) by sonography (US) in professional water polo players with shoulder impingement syndrome (SIS).

Methods Twenty-four professional water polo players (mean age: $22.13 \pm 3.34$ ) with SIS were assigned to one of 2 different treatment interventions: Group (1) MSM: a series of fast accelerations in the upward direction was applied to the upper limb that's connected to a spring through a metal plate with a ring. The ring was linked to a pulley system that was submitted to forces acting in the opposite direction (added mass). Group (2) Simple traction: the series of fast accelerations were performed without the springs. Pain intensity, Yocum and Hawkins tests for SIS, Neer's impingement sign, range of motion, muscle strength and shoulder US were assessed. The examination was performed before, immediately after and 30 days after each treatment to study the US width of subacromial-subdeltoid bursa (SSB), thickness of supraspinatus (ST), long biceps tendons (LBT); hypoechoic halo of surrounding the long biceps (LBH) and subscapular tendons (STH); width of acromio-clavicular joint capsule (ACJ) and the distance between bone heads (ACD). Impingement sign (IS) was evaluated by dynamic examination.

Results Immediately after treatment with MSM, pain was much reduced $(p=0.002)$; Yocum and Hawkins tests were decreased ( $p=0.008, p=0.031)$; Neer's impingement sign was negative; range of motion and muscle strength were increased. US showed that the following parameters were significantly reduced: SSB $(p=0.001), \operatorname{LBT}(p=0.014), \operatorname{LBH}(p=0.014)$, SSH $(p=0.002)$, ACJ $(p=0.004)$, ACD $(p=0.001)$. IS was no more detected. After 30 days, the improvement of clinical and US findings was maintained. In the control group, after simple traction, no clinical amelioration of US parameters was found immediately after the procedure.

Conclusion These data show that MSM could be significantly and rapidly effective against pain and the loss of function due to shoulder impingement in water polo players.
\end{abstract}

Keywords Muscle shortening manoeuvre (MSM) - Shoulder impingement · Sonographic exam · Water polo players · Rehabilitation therapy

Diego Longo

diego.longo@unifi.it

1 Department Experimental and Clinical Medicine, University of Florence, Florence, Italy

2 Sezione di Scienze Fisiologiche, Dipartimento di Medicina Sperimentale e Clinica, Università degli Studi di Firenze, Viale Morgagni 63, 50100 Florence, Italy
3 Department of Surgery and Transplantation, Academic Hospital of Santa Maria della Misericordia Udine, University of Udine, Udine, Italy

4 Unit of Immunology, Rheumatology, Allergy and Rare Diseases (UnIRAR), IRCCS San Raffaele Hospital, Milan, Italy

5 Division of Rheumatology AOUC, Florence, Italy 


\section{Introduction}

We know that Shoulder Impingement Syndrome (SIS), which is a multifactorial condition, is the most common cause of shoulder pain. [1]. Usually, its etiology is due to the modification of intrinsic and extrinsic mechanisms of the rotator cuff eliciting or eventually a damage of the shoulder structures [2,3]. External mechanical compression of the anatomical structures within the subacromial space is involved in SIS generation [3]. The bone alterations of the acromion and the acromio-clavicular joint (ACJ) and the thickness of the coracoacromial ligament may be considered extrinsic factors in SIS. In addition, the consequence of an unbalanced action of shoulder muscles with loss of central position of the humeral head may be related to SIS [4-8]. This causes a deficit in the muscular performance of the rotator cuff and the scapular muscles $[2,3,9]$. It is suggested that muscle unbalance may be removed by muscle shortening manoeuvre (MSM) $[10,11]$ which has been shown before to be an effective therapeutic application in SIS [12, 13]. In fact, it has been evidenced that MSM is the main outcome of increased muscle strength. This manoeuvre increases serratus anterior muscle strength, stabilizing and balancing the joint with pain relief [12-15]. Various sports activities can cause SIS from prolonged and intense activities. Water polo is an "open skill" and "overhead" sport where the shoulder elevation is continuously above $90^{\circ}$ with repeated and frequent rotational movements. These movements may provoke pain, injury of glenoid labrum and SIS reducing the performance during activity [16-20]. Water polo has cyclic movements and coordination complexes with frequent changes in direction, position and contraction intensity of the upper and the lower limb. These complex activities, with a specific dynamic shot, are essentially characterized by five functional steps (preparation/loading/acceleration/deceleration/follow through). The continuous repetition of the activity progressively determines a chronic microtrauma of the shoulder evolving into SIS.

The aim of our work was to evaluate, in professional water polo players with SIS, the effects of MSM on shoulder pain and muscle strength and to compare the shoulder modifications obtained with MSM by the means of US parameters.

Our data clearly shows that MSM obtains rapid improvement in SIS and maintains its effects after a month.

\section{Materials and methods}

24 out of 68 professional players of the Florence water polo team ("Firenze Pallanuoto") [14 male and 10 female; mean age: $22.13 \pm 3.34$ (range 17-30)], with SIS, were enrolled in the study. Patients were included in the study if they fulfilled the following diagnostic criteria: players with chronic shoulder pain, pain provoked by abduction of shoulder with painful arc, positive Neer's impingement sign $[6,21]$ and positive Yocum and Hawkins tests [22, 23]. Exclusion criteria were: ex-players for at least 1 year, recent acute trauma $(<2$ months), physiotherapy treatment for at least 1 month and NSAIDs intake two days before inclusion in this study. The study was approved by the local ethic committee (CEA 14900_spe) and all players signed the informed consent.

Enrolled players were randomly assigned to one of 2 different treatment interventions. The first group of 14 players was treated with MSM: a series of fast accelerations in the upward direction was applied to the upper limb that's connected to a spring through a metal plate with a ring. The ring was linked to a pulley system that was submitted to forces acting in the opposite direction (added mass). The second control group of 10 players was treated with a simple traction only: the series of fast accelerations were performed without the springs. Pain intensity, Yocum and Hawkins tests, Neer's impingement sign, range of motion and muscle strength (Jtech Company dynamometer) were evaluated. The examination was performed before, immediately after and 30 days after each treatment. Pain intensity was assessed by a numerical rating scale (NRS) in a range of $0-10$ points; range of motion (ROM) was evaluated in passive (PROM) and active (AROM) manner during intra and extra rotation (IR, ER), flexion and abduction of the shoulder (FS, AS) with the patient lying by a digital goniometer (Jtech Commander Echo). The value of ROM was considered reduced when $<90^{\circ}$ during intra ed extra rotation; $<180^{\circ}$ during flexion and abduction. Muscle strength was measured in the movement of flexion (MS FS), abduction (MS AS) with sitting subject, intra and extra rotation (MS IR, MS ER) with prone subject, by digital dynamometer (Jtech Commander Echo). The mean values were expressed in Newton. Sonography (US) was performed by a sonographer (DM) blinded to the results of clinical evaluation.

\section{Sonographic exam}

US was performed, as reported in earlier studies [13, $14,24,25]$ in all players before, immediately after and 30 days after each treatment with equipment ESAOTE 
MyLab 70 X-Vision with linear probe $8-16 \mathrm{MHz}$. The interobserver $(\kappa=0.88)$ and intraobserver $(\kappa=0.98)$ agreement was assessed. The following sonographic features were evaluated: (1) width of the subacromial-subdeltoid bursa (SSB); (2) thickness of supraspinatus (ST) and long biceps tendons (LBT); (3) hypoechoic halo surrounding the long biceps (LBH) and subscapularis tendons (STH); (4) width of acromioclavicular joint (ACJ) capsule and the distance between bone heads (ACD). Impingement (IS) was studied by dynamic examination with evaluation of coracoacromial ligament position. The glenoid labrum was also examined [26, 27].

\section{Muscle shortening manoeuvre}

This manoeuvre, introduced by Grimaldi [10-13, 15], causes dynamic stretching. It is associated with a sudden shortening of the muscles responsible for shoulder protraction and retraction. MSM was performed on each player of the first group.

Participants were positioned in a supine position with the shoulder joint flexed to $90^{\circ}$, the elbow in extension and with a clenched fist.

A first bandage was applied to a patient to protect the skin (using simple absorbent paper) starting from the proximal third of the shoulder, covering the hand. An eyelet plate was then placed on the proximal phalanges (second to fifth finger). The plate was anchored through a carabiner hook to the elastic elements, from one to three springs, depending on the weight of the limb. The springs, $50 \mathrm{~cm}$ long and $2 \mathrm{~cm}$ in diameter, were in turn connected to a pulley through a rope. The whole set was fixed with adhesive tape. In correspondence of the gleno-humeral joint, a spring with a parallelepiped weight (of $4 \mathrm{~kg}$ ) was positioned, to increase the elastic return of the spring.

The physiotherapist, acting on the rope, applied manually stresses by moving the scapula in the direction of protraction, in a rhythmic way, with a frequency of about $2 \mathrm{~Hz}$ for a duration of $10 \mathrm{~min}$. In each subject, the manoeuvre was performed only once.

\section{Simple traction}

The simple traction was performed on each player of the second group.

It consisted of a simulation of MSM, offering all its characteristics, excluding the presence of the elastic elements (springs). In this way, the experimenters set out to perform a solicitation quite comparable to passive mobilization of the shoulder in protraction. As with MSM, the treatment duration was set at $10 \mathrm{~min}$ and each participant was treated only once.

\section{Statistical analysis}

IBM SPSS Statistics version 26 software was used for the statistical analysis.

In order to verify the normality of the data distribution, a Kolmogorov-Smirnov test was adopted.

The paired samples $t$-test was used to check the statistical significance of the differences within the groups for normally distributed variables. The independent samples $t$-test was adopted to verify the statistical significance of the differences between groups for the same kind of variables.

The Wilcoxon test and the Mann-Whitney test were used for the same purposes, for variables not distributed according to the normal.

For dichotomous variables, the Chi-squared test was used for differences within the groups while the McNemar test for those between groups.

\section{Results}

Results are shown in Table 1, 2 and 3.

In group $1 \mathrm{MSM}$ induced an improvement of the pain at T1 $(p=0.006)$ and T2 $(p=0.02)$, of the Yocum test at T1 $(p=0.063)$ and T2 $(p=0.008)$, of the Hawkins test at $\mathrm{T} 1(p=0.125)$ and T2 $(p=0.031)$, of the passive ROM at $\mathrm{T} 1$ (flexion $p=0.030$; abduction $p=0.013$, internal rotation $p=0.463$ ) and T2 (flexion p 0.001; abduction p 0.035, internal rotation 0.002 ), of the active ROM at T1 (flexion $p=0.085$, abduction $p=0.028$, internal rotation $p=0.925)$ and T2 (flexion $p$ 0.002; abduction p 0.016, internal rotation 0.001) (Table 2). US findings showed in group 1, after MSM, a decrease of thickness of SSB at T1 $(p=0.01)$, and $\mathrm{T} 2(p=0.001)$, of LBT at T1 $(p=0.635)$ and T2 $(p=0.014)$; a decrease of LBH at T1 $(p=0.008)$ and T2 $(p=0.014)$, of STH at T1 $(p=0.08)$ and T2 $(p=0.002)$; a decrease in width of ACJ at T1 $(p=0.005)$ and T2 $(p=0.004)$; a reduction of bone heads ACD at T1 $(p=0.002)$ and T2 $(p=0.001)$. IS was absent in 12 out of 14 subjects, (Table 3 ).

In group 2 the simple traction did not show a significant change in all subjects.

Table 1 Characteristics of the patients

\begin{tabular}{lll}
\hline & Group 1 & Group 2 \\
\hline Age & $21.71 \pm 2.99$ & $22.7 \pm 3.86$ \\
Female & 6 & 4 \\
Male & 8 & 6 \\
Shoulder R & 13 & 10 \\
Shoulder L & 1 & 0 \\
IS & 14 & 10 \\
\hline
\end{tabular}




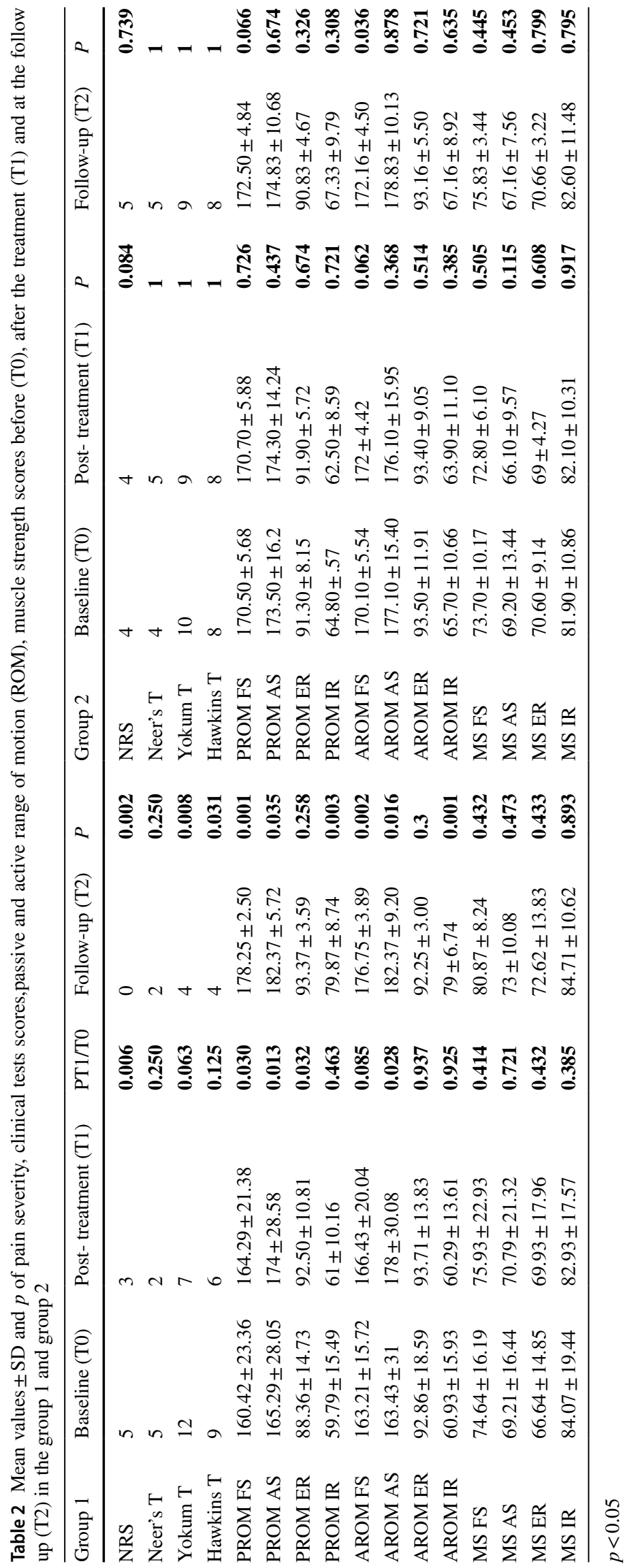




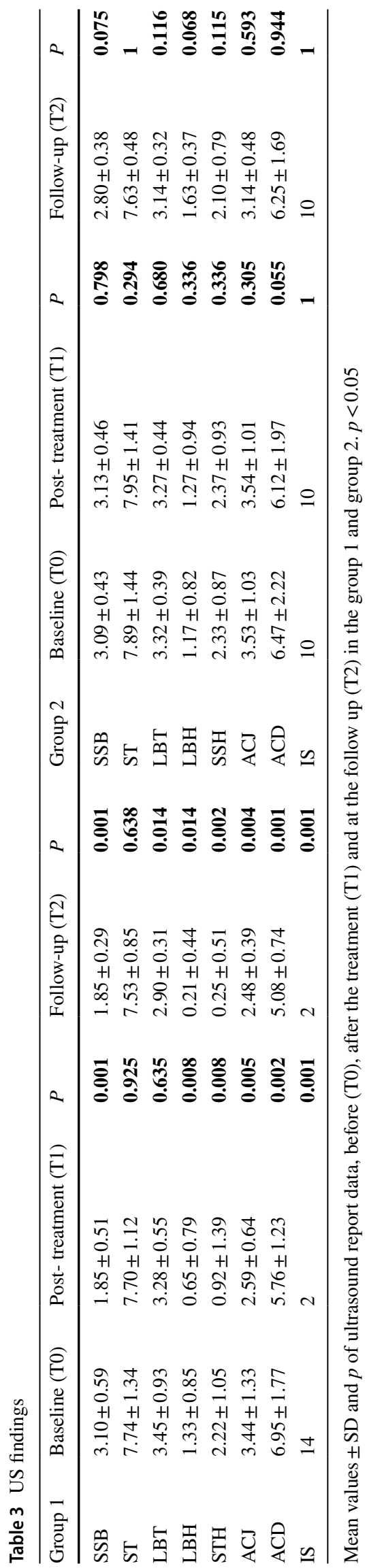

Table 4 Groups Comparison $p$ values of mean/median comparison of pain severity, clinical tests scores, passive and active range of motion (ROM), muscle strength scores before (T0), after the treatment (T1) and at the follow up (T2) between groups

\begin{tabular}{llll}
\hline Group 1/Group 2 & Baseline (T0) & $\begin{array}{l}\text { Post- treat- } \\
\text { ment (T1) }\end{array}$ & Follow-up (T2) \\
\hline NRS & 0.690 & 0.572 & 0.001 \\
Neer's T & 0.831 & 0.058 & 0.058 \\
Yokum T & 0.212 & 0.040 & 0.003 \\
Hawkins T & 0.633 & 0.069 & 0.013 \\
PROM FS & 0.277 & 0.618 & 0.002 \\
PROM AS & 0.482 & 0.558 & 0.036 \\
PROM ER & 0.659 & 0.680 & 0.212 \\
PROM IR & 0.100 & 0.860 & 0.004 \\
AROM FS & 0.216 & 0.977 & 0.006 \\
AROM AS & 0.363 & 0.240 & 0.054 \\
AROM ER & 0.638 & 0.791 & 0.512 \\
AROM IR & 0.617 & 0.481 & 0.002 \\
MS FS & 0.517 & 0.725 & 0.038 \\
MS AS & 0.998 & 0.525 & 0.137 \\
MS ER & 0.596 & 0.618 & 0.274 \\
MS IR & 0.753 & 0.895 & 0.647 \\
\hline
\end{tabular}

$p<0.05$

Table 5 Groups Comparison $p$ values of ultrasound report data,before (T0), after the treatment (T1) and at the follow up (T2) between groups

\begin{tabular}{llll}
\hline Group 1/Group 2 & Baseline (T0) & $\begin{array}{l}\text { Post- treat- } \\
\text { ment (T1) }\end{array}$ & Follow-up (T2) \\
\hline SSB & 0.680 & 0.000 & 0.000 \\
ST & 0.883 & 0.660 & 0.301 \\
LBT & 0.792 & 0.702 & 0.046 \\
LBH & 0.906 & 0.079 & 0.000 \\
STH & 0.596 & 0.003 & 0.000 \\
ACJ & 0.639 & 0.017 & 0.005 \\
ACD & 0.538 & 0.747 & 0.067 \\
IS & 0.388 & 0.000 & 0.000 \\
\hline
\end{tabular}

$p<0.05$

Comparison between the groups shows better efficacy of MSM than simulated traction of NRS at T2 $(p=0.001)$, the Yocum test at T1 $(p=0.040)$ and T2 $(p=0.003)$, the Hawkins test at $\mathrm{T} 2(p=0.013)$, passive ROM at T2 (flexion $p=0.002$; abduction $p=0.036$; internal rotation $p=0.004$ ), active ROM at T2 (flexion $p=0.006$; internal rotation $p$ 0.002) and strength muscle at T2 (flexion $p=0.038$ ) (Table 4).

Comparison of US results between groups also showed some statistically significant differences about SSB thickness at T1 $(p=0.000)$ and T2 $(p=0.000)$, LBT thickness at T2 $(p=0.046), \mathrm{LBH}$ at T2 $(p=0.000), \mathrm{SSH}$ at T1 
Fig. 1 a SSB thickness before MSM (T0); b SSB thickness after MSM (T1); c the distance between the bony heads of the ACD before MSM; $\mathbf{d}$ the distance between the bony heads of the ACD after MSM

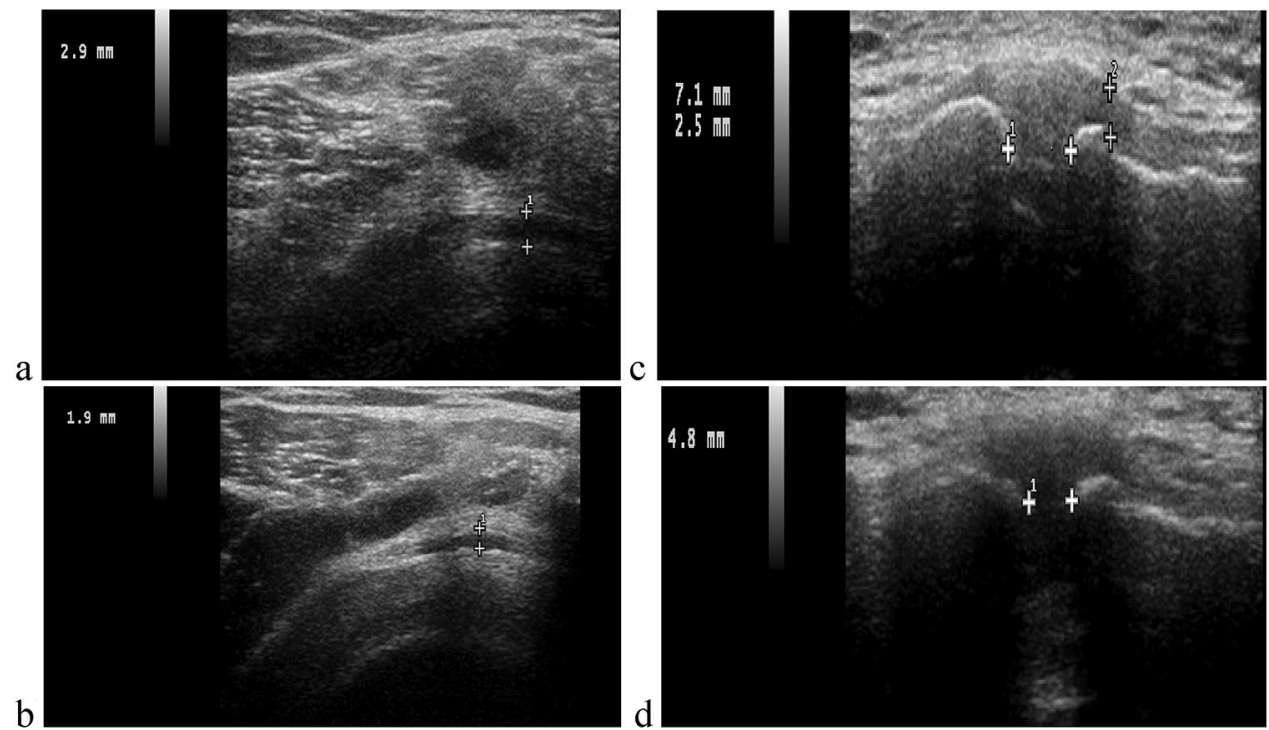

$(p=0.003)$ and T2 $(p=0.000)$ and IS at T1 $(p=0.000)$ and $\mathrm{T} 2(p=0.000)$ (Table 5).

\section{Discussion}

The main difficulty in the sport of water polo is finding the ideal position to make an effective shot. Therefore, the importance of a stable shoulder is obvious. In fact, during the preparation phase, the shoulder is maintained in an abducted position due to the activation of the medial and anterior part of the deltoid, of the supraspinatus and of the clavicular part of the pectoralis major. In the loading phase, an excessive articular excursion is determined, which involves an over distension of the capsular-ligamentous component and a consequent relative instability of the glenohumeral joint [28]. MSM plays, as demonstrated in earlier studies, an important function in shoulder stabilization, as it results, in rapid self-centering of the head of the humerus $[12,13]$. Our results showed that MSM is able to remove impingement, even in water polo players, as confirmed by US measurements (Table 3 ). Moreover, the data of this study showed other improvements in group 1 : a significant difference of pain both at T1 $(p=0.006)$ and at T2 $(p=0.02)$ and of the passive and the active ROM at $\mathrm{T} 1$ and at $\mathrm{T} 2$ as reported in Table 2. These results were also confirmed by the comparison between the two groups (Table 4). No athlete had symptoms consistent with a glenoid labrum injury and this was confirmed with US at time T0. In all athletes in group 1, US detected a marked improvement in the considered ultrasound parameters with a significant difference in both $\mathrm{T} 1$ and $\mathrm{T} 2$, as reported in Table 3. In SIS, the thickness of SSB appears increased. US findings confirm this at T0 (Fig. 1). It is important to point out that in water polo players of the first group, US, immediately after MSM, showed reduction of the bursa with a significant difference at T1 $(p=0.01)$ and at T2 $(p=0.001)$ (Table 3). A similar result was obtained when comparing the two groups (Table 5). In our results, we found an unexpected finding related to the distance between the bony heads of the ACD (Fig. 1). In fact, this distance appeared markedly and significantly reduced at $\mathrm{T} 1(p=0.002)$ and $\mathrm{T} 2(p=0.001)$ if compared to T0 in water polo players in group 1 . In conclusion, our results showed the efficacy of MSM with a persistent amelioration at $\mathrm{T} 2$ and the improvement of water polo players performance during the follow-up period. It should be noted that the effectiveness of MSM is improved when comparing the two groups with simple traction.

PERSPECTIVE: There are many data in the literature regarding the Grimaldi manoeuvre in the treatment of SIS $[12,13]$. This manoeuvre has the particularity of being able to be performed in a single session with immediate and persistent recovery of the functionality of the shoulder with improvement of pain symptoms [10-13]. The use of the ultrasound examination allows you to be able to appropriately detect shoulder disorders and perform follow-up in overhead athletes $[24,25]$. In the present study, it was shown that water polo players could benefit from physiotherapy manoeuvre to improve sports performance. In the follow up of these athletes, the ultrasound examination was useful. In future studies, the manoeuvre could be applied to a wider casistic, including athletes from other overhead disciplines.

Funding The authors did not receive support from any organization for the submitted work. 


\section{Declarations}

Conflict of interest The authors have no relevant financial or non-financial interests to disclose.

Ethical approval The study was performed in accordance with the ethical standards as laid down in the 1964 Declaration of Helsinki and its later amendments or comparable ethical standards.

Consent to participate Informed consent was obtained from the individual.

Consent to publish The participants have consented to the submission of the original article to the journal.

Data availability All data generated or analyzed during this study are included in this published article.

\section{References}

1. Juel NG, Natvig B (2014) Shoulder diagnoses in secondary care, a one year cohort. BMC Musculoskelet Disord 15(89):1-8

2. Michener LA, McClure PW, Karduna AR (2003) Anatomical and biomechanical mechanisms of subacromial impingement syndrome. Clin Biomech 18:369-379

3. Seitz AL, McClure PW, Finucane S et al (2011) Mechanisms of rotator cuff tendinopathy: intrinsic, extrinsic, or both? Rev Clin Biomech 26:1-12

4. Van der Windt DA, Koes BW, De Jong BA et al (1995) Shoulder disorders in general practice: incidence, patient characteristics, and management. Ann Rheum Dis 54:959-964

5. Brox JI (2003) Shoulder pain. Best Pract Res Clin Rheumatol $17: 33-56$

6. Beltran LS, Nikac V, Beltran J (2012) Internal impingement syndromes. Magn Res Imaging Clin N Am 20:201-211

7. Haahr JP, Ostergaard S, Dalsdgaard J et al (2005) Exercises versus arthroscopic decompression in patients with subacromial impingement: a randomized, controlled study in 90 cases with a one year follow up. Ann Rheum Dis 64:760-764

8. Papadonikolakis A, Mckenna M, Warme W et al (2011) Published evidence relevant to the diagnosis of impingement syndrome of the shoulder. J Bone Jt Surg Am 93:1827-1832

9. Ludewig PM, Braman JP (2011) Shoulder impingement: biomechanical considerations in rehabilitation. Man Ther 16(1):33-39

10. Grimaldi L, Lippi P, Marri P et al (1986) Evoking of absent motor components in CNS lesions. Editori Giardini, Pisa, p 88

11. Crippa A, Cardini R, Pellegatta D et al (2004) Effects of sudden passive muscle shortening according to Grimaldi's method on patients suffering from multiple sclerosis: a randomized controlled trial. Neurorehabilitation Neural Repair 18(1):47-52

12. Melchiorre D, Lippi P, Marchi A et al. (2018) Muscle shortening maneuver and not topical anti-inflammatory therapy is effective in reducing the width of subacromial-subdeltoid bursa in shoulder impingement syndrome. Proc. SPIE 10582, laser Florence 2017: advances in laser medicine, p. 105820B. Doi: https://doi.org/10. $1117 / 12.2319694$

13. Melchiorre D, Maresca M, Bracci R et al (2014) Muscle shortening manoeuvre reduces pain and functional impairment in shoulder impingement syndrome: clinical and ultrasonographic evidence. Clin Exp Rheumatol 32(1):5-10

14. Slemenda C, Brandt KD, Heilman DK et al (1997) Quadriceps weakness and osteoarthritis of the knee. Ann Int Med 127:97-104

15. Longo D, Longo L, Lippi P et al (2017) Effects of laser therapy and Grimaldi's muscle shortening maneuver on motor control of subjects with incomplete spinal cord injuries. Laser Ther 26(3):203-209

16. Hams A, Evans K, Adams R et al (2019) Epidemiology of shoulder injury in sub-elite level water polo players. Phys Ther Sport 35:127-132

17. Hams A, Kerry E, Adams R et al (2019) Shoulder internal and external rotation strength and prediction of subsequent injury in water-polo players. Scand J Med Sci Sports 29:1414-1420

18. Hams A, Evans K, Adams R et al (2019) Reduced shoulder strength and change in range of motion are risk factors for shoulder injury in water polo players. Phys Ther Sport 40:231-237

19. Miller AH, Evans K, Adams R et al (2019) Shoulder injury in water polo: a systematic review of incidence and intrinsic risk factors. J Sci Med Sport 21(4):368-377

20. Stromberg JD (2017) Care of water polo players. Curr Sports Med Rep 16(5):363-369

21. Neer CS (1983) Impingement lesions. Clin Orthop Relat Res 173:71-77

22. Yocum LA (1983) Assessing the shoulder: history, physical examination, differential diagnosis, and special tests used. Clin Sports Med 2(2):281-289

23. Hawkins RJ, Kennedy JC (1980) Impingement syndrome in athletes. Am J Sports Med 8(3):151-158

24. Farin PU, Jarom H, Harju A et al (1990) Shoulder impingement syndrome: sonographic evaluation. Radiology 176:845-849

25. Galluccio F, Bellucci E, Porta F et al (2017) The waterpolo shoulder paradigm: results of ultrasound surveillance at poolside. BMJ Open Sport Exerc Med 3(1):e000211

26. Middleton WD, Reinus WR, Totty WG et al (1986) Ultrasonographic evaluation of the rotator cuff and biceps tendon. J Bone Surg (Am) 68:440-450

27. Crass JR, Craig EV, Feinberg SB (1987) The hyperextended internal rotation view in rotator cuff ultrasonography. J Clin Ultrasound 15:416-420

28. Matzkin E, Suslavich K, Wes D (2016) Swimmer's shoulder: painful shoulder in the competitive swimmer. J Am Acad Orthop Surg 24(8):527-536

Publisher's Note Springer Nature remains neutral with regard to jurisdictional claims in published maps and institutional affiliations. 